\title{
Perfil de Aprendizaje y Rendimiento Académico en una Asignatura de Química en Modalidad a Distancia y Presencial en dos Programas de Ingeniería
}

\author{
Diofanor Acevedo*, Diego F. Tirado y Piedad M. Montero \\ Universidad de Cartagena, Facultad de Ingeniería, Programa de Ingeniería de Alimentos, Grupo de \\ Investigación NUSCA, Avenida el Consulado, Calle 30 No. 48-152. Cartagena, Bolívar-Colombia \\ (e-mail: diofanor3000@gmail.com)
}

* Autor a quien debe ser dirigida la correspondencia

Recibido Ene. 27, 2014; Aceptado Abr. 4, 2015; Versión final May. 13, 2015, Publicado Dic. 2015

\section{Resumen}

Se analizó el aprendizaje y rendimiento académico de estudiantes que cursaron la asignatura química en las modalidades distancia y presencial en los programas Ingeniería Electrónica y Electromecánica de una Institución de Educación Superior con sede en Cartagena de Indias (Colombia). De 113 estudiantes, $36.28 \%$ pertenecían a la modalidad presencial y $63.71 \%$ a la de distancia. Se empleó el cuestionario VARK para identificar los estilos visual, auditivo, lecto-escritor y kinestésico, y se recopilaron las calificaciones de una plataforma institucional. Se construyó una base de datos, y aplicaron pruebas para comparar resultados. Predominó el estilo kinestésico $(32.5 \%)$ en ambas modalidades $(p<0.05)$. Los rendimientos fueron $88.6 \%$ para distancia y $78.4 \%$ en presencial $(p<0.05)$. A los estudiantes se les facilitó el aprendizaje empírico, siendo la modalidad de distancia la que se adaptó mejor a esta metodología. No hubo correlación entre los estilos de aprendizaje y rendimiento académico en ambas modalidades ( $p>0.05)$.

\section{Profile Learning and Academic Performance in Course of Chemistry in the Online and Face-to-Face Modes in two Engineering Programs}

\begin{abstract}
Learning and academic performance of students who studied a chemistry course in distance and face-toface modalities in Electronics and Electromechanical Engineering programs in a higher education institution based in Cartagena de Indias (Colombia) was analyzed. Of 113 students, $36.28 \%$ belonged to the modality and $63.71 \%$ for distance. The VARK questionnaire was used to identify the visual, auditory, and kinesthetic reading-writing styles, and qualifications of an institutional platform were collected. A database was constructed and tests were applied to compare results. Kinesthetic was the predominated style $(32.5 \%)$ in both modes $(p<0.05)$. The yields were $88.6 \%$ for distance students and $78.4 \%$ for students in classroom $(p<0.05)$. Students were provided with empirical learning, being the distance mode the one that best adapted to this methodology. There was no correlation between learning styles and academic performance in both modes $(p>0.05)$.
\end{abstract}




\section{INTRODUCCIÓN}

El ser humano posee mecanismos especiales que le permiten aprender y representar la realidad, no solamente para atender sus necesidades básicas, sino también para dar respuesta a las demandas del ambiente en que se desenvuelve (Aguilera, 2012). Autores como López-Aguado, (2011), y Gutiérrez et al., (2012), afirman que cada estudiante asimila de manera distinta a los demás, utilizando diversas formas y velocidades que cambian durante el proceso formativo. Conceptualmente los estilos de aprendizaje se entienden como variables personales que, en términos de inteligencia y personalidad, explican las diferentes formas de abordar, planificar y responder ante las demandas del aprendizaje y se manifiestan a través de un patrón conductual (Ventura, 2011; Bahamón et al., 2013).

Como una forma de buscar respuestas sobre la calidad de la enseñanza y la manera de aprender de las personas, en la década de los ochenta Neil Fleming, construyó el cuestionario Visual, Aural, Read/write and Kinesthetic (VARK), que evalúa cuatro estilos de aprendizaje: visual, auditivo, lecto/escritor y kinestésico, los cuales se miden a través de trece preguntas con cuatro opciones de respuesta (Fleming, 2007). Este ha sido aplicado en numerosos estudios, ya que es de fácil manipulación y entrega información rápida sobre la forma de aprendizaje de los individuos (León-Castañeda et al., 2010). La preferencia visual se refiere a adquirir la información a través de imágenes, representaciones gráficas que incluyan cuadros, flechas o símbolos. En la preferencia auditiva predomina la capacidad de escuchar facilitándose el aprendizaje en conferencias y diálogos. En la dimensión lectura/escritura se percibe más fácilmente la información mostrada en palabras escritas. En la preferencia kinestésica predomina el aprendizaje práctico, se facilita el aprender haciendo y experimentando con el mundo. Valencia et al., (2012), afirma que en esta modalidad se utilizan simultáneamente varios sentidos y se caracteriza por una fuerte relación con el ambiente, y además indica que es la dimensión más estructurada y compleja.

Sepúlveda-Carreño et al., (2011), aseguran que existe una relación positiva entre los estilos de aprendizaje y el rendimiento académico. Lo cual se relaciona con lo reportado por Herrera, y Zapata, (2012), quienes aseguran que los estilos de aprendizaje son susceptibles de mejorarse cuando a los estudiantes se les enseña según sus propias capacidades, y que esta situación, puede llegar a garantizar procesos de enseñanza-aprendizaje de alta calidad. Así mismo Esguerra y Guerrero, (2010) encontraron evidencias de que presentar la información mediante distintos estilos lleva a una instrucción y asimilación más efectiva. Sin embargo delimitar el concepto y ámbito de aplicación del término rendimiento académico no ha sido tarea fácil, dado el carácter complejo y multidimensional de esta variable del área educativa (Blumen et al., 2011).

Montero-Rojas et al., (2007) define el concepto de rendimiento académico como el resultado del aprendizaje, suscitado por la intervención pedagógica del docente, y producido en el estudiante. No es el producto analítico de una única aptitud, sino más bien el resultado sintético de una suma (nunca bien conocida) de elementos que actúan en y desde la persona que aprende, tales como factores institucionales, pedagógicos, psicosociales y sociodemográficos. El indicador más utilizado para el rendimiento académico ha sido la calificación cuantitativa, la cual, si es equilibrada y legítima, será el reflejo de un determinado aprendizaje del estudiante en el contexto educativo (Blumen et al., 2011). La forma más directa de establecerla es a través de exámenes o pruebas de medición, que pueden o no tener falencias de elaboración, ya que la forma de evaluar la decide el docente, en la mayoría de los casos con criterios personales, por lo que se dificulta la comparación dentro de un mismo centro universitario y con otros centros educativos (Bahamón et al., 2013).

Las críticas y lo discutible de las calificaciones en la mayoría de las investigaciones se refieren a esta medida como reflejo del desempeño, siendo además considerada en los requisitos de becas, promoción a niveles educativos superiores y obtención de empleos, como el principal indicador de los resultados académicos de un alumno (Willcox, 2011). No obstante, se debe tener en consideración que las calificaciones en sí mismas ostentan un valor relativo como cuantía del rendimiento académico, ya que no existe un criterio estandarizado para todos los centros de educación, especialmente universitarios (Li et al., 2010). En este trabajo se utilizó la calificación de las actividades asignadas a un grupo de estudiantes que cursaron la asignatura de química en dos programas de Ingeniería de una Institución de Educación Superior (IES) con sede en la ciudad de Cartagena de Indias (Colombia), para cuantificar el rendimiento académico de los mismos. Aunque se reconocen sus limitaciones (Willcox, 2011), se considera que dentro de los mecanismos para medir el rendimiento éstas siguen siendo las más efectivas. Teniendo en cuenta lo anterior, el objetivo de este trabajo fue analizar el perfil de aprendizaje y el rendimiento académico de los estudiantes de tercer semestre que cursaron la asignatura química a distancia y presencial en los programas de Ingeniería Electrónica y Electromecánica de una IES con sede la ciudad de Cartagena de Indias durante los periodos 2012-1 al 2013-2. 


\section{MATERIALES Y MÉTODOS}

Se realizó un estudio descriptivo no experimental y de corte secuencial. La muestra estuvo conformada por un total de 113 estudiantes que cursaron la asignatura de química en dos modalidades: a distancia $(63,71 \%)$, los cuales fueron del programa Ingeniería Electromecánica; y presencial $(36,28 \%)$ matriculados en el programa Ingeniería Electrónica de la IES con sede en Cartagena de Indias, durante los periodos académicos de 2012-1 al 2013-2. Es importante destacar que todos los estudiantes matriculados en la asignatura durante el periodo analizado fueron hombres. La edad para los estudiantes de la modalidad presencial osciló de 17 a 25 años, con una media de 21,19 $\pm 0,38$ años. Mientras que para los estudiantes de la modalidad distancia varió de 28 a 55 años con una media de $32,41 \pm 0,38$ años. La mayoría de ellos, provenientes de familias de estrato socioeconómico tres, seguido del estrato socioeconómico dos y cuatro, en tanto, los estratos menos representados fueron el uno y el seis.

\section{Evaluación de los estilos de aprendizajes con el cuestionario VARK}

Al inicio de cada período se realizó un diagnóstico sobre los perfiles de aprendizaje de cada grupo que cursó la asignatura de química, en modalidad presencial como a distancia. Se utilizó la versión traducida del cuestionario VARK, desarrollado y validado por Neil Fleming en 1987. Este formato permitió identificar los estilos Visual, Auditivo, Lecto-escritor y Kinestésico, y ha sido aplicado en investigaciones importantes como la realizada por León-Castañeda et al., (2010) en la identificación de los perfiles de aprendizaje de los estudiantes y profesores del programa de medicina en la Universidad nacional autónoma de México. El instrumento fue contestado por un total de 41 estudiantes de Ingeniería Electrónica y 72 de Electromecánica. Las respuestas se recopilaron, procesaron y se dividieron en dos categorías: baja $(\leq 7,9)$ y alta $(\geq 8,0)$.

\section{Descripción de la asignatura de química}

La asignatura está ubicada en tercer semestre dentro de los planes curriculares de ambas carrera y es teórico-práctica, con la realización de cuatro prácticas de laboratorio por modalidad durante el semestre. Fue dictada por el mismo docente, siguiendo los lineamientos estipulados en el planeador de contenidos de la disciplina de química, documento elaborado por la vicerrectoría académica de la IES. En este se hallaron los horarios, los temas a tratar en el aula de clase, las fuentes de información bibliográficas, los objetivos específicos, así como las estrategias para el desarrollo de las competencias y habilidades que los estudiantes tuvieron que alcanzar para aprobar esta área de conocimiento. Cabe resaltar que el Instrumento de recopilación de las calificaciones de los estudiantes y la metodología de enseñanza aplicada en la asignatura de química fue implementado por un docente, y no refleja la perspectiva de los autores.

\section{Metodología de enseñanza aplicada en la asignatura de química}

Se evaluaron cuatro cohortes durante el semestre, en ambas modalidades, con los respectivos porcentajes: $20 \%, 20 \%, 30 \%, 30 \%$. La escala de calificación fue de 0,0 a 5,0 dónde el mínimo para aprobar la asignatura fue de 3,0. El período académico semestral estuvo conformado por 16 semanas y un crédito presencial implicó una hora en el aula de clase y dos de forma independiente por parte del estudiante. Mientras que en la modalidad a distancia, la relación fue de una hora en el aula y cinco fuera de ella, tal como se muestra en la Tabla 1.

Tabla 1: Relación créditos académicos presencial y a distancia en la IES

\begin{tabular}{|c|c|c|c|c|}
\hline \multirow{2}{*}{ Un crédito académico } & \multicolumn{4}{|c|}{ Horas de trabajo a la semana asignatura química } \\
\cline { 2 - 5 } & Relación & Aula de clase & Independiente & Total \\
\hline Presencial & $1 / 2$ & 4 & 8 & 12 \\
\hline Distancia & $1 / 5$ & 2 & 10 & 12 \\
\hline
\end{tabular}

En la modalidad presencial se dictaron clases magistrales, mientras que en distancia se emplearon tutorías dirigidas por el docente, donde el estudiante tuvo la iniciativa. A su vez se utilizó la aplicación educativa virtual Moodle en su versión 2.2, la cual fue de gran utilidad, ya que ayudó a crear comunidades de aprendizaje en línea, respaldando la interacción grupal, al mismo tiempo que permitió la conversación privada entre los estudiantes y entre estos con el docente. Se evaluaron las mismas actividades académicas por cohorte para ambas modalidades con los siguientes porcentajes: Examen teórico al inicio de cohorte $(20 \%)$, Talleres independientes $(10 \%)$, Proyecto de aula $(20 \%)$, Seminarios con artículos de investigación (10\%), Prácticas de laboratorio (10\%) y Examen teórico al final de cohorte $(30 \%)$. 
Todas las evaluaciones fueron del tipo "razonamiento" y no aquéllas en las que se piden definiciones textuales, que por lo general terminan midiendo sólo la habilidad memorística de los estudiantes. En la modalidad presencial las prácticas de laboratorios fueron dirigidas por el profesor en las instalaciones correspondientes, quien entregó durante las semanas previas guías sobre los procedimientos a seguir, las cuales se explicaron durante las respectivas prácticas. En la modalidad a distancia el docente envió al correo electrónico de los estudiantes las respectivas guías, y adicional a ello videos ilustrativos sobre la temática a evaluar. Estos videos fueron clasificados y bajados de la página YouTube, y luego analizados por los estudiantes, quienes a su vez elaboraron un protocolo sobre los pasos a seguir el día de cada práctica. Al final efectuaron los respectivos ensayos experimentales.

\section{Instrumentos para recopilar las calificaciones de los estudiantes}

Los datos de los estudiantes como: edad, género, naturaleza del colegio de procedencia, calificaciones académicas y otros de la hoja de vida, fueron extraídos de la Plataforma Educativa de Registro Académico de la IES, la cual es de acceso exclusivo para los docentes de las distintas facultades de dicha institución, y en la cual se encuentran las calificaciones de los estudiantes por disciplinas, los horarios, códigos y salones asignados, en este caso, de los que cursaron la asignatura química en los programas Ingeniería Electrónica, y Electromecánica en las modalidades presencial y a distancia respectivamente.

\section{Análisis de datos}

Después de obtener la información sobre los perfiles de aprendizaje y las calificaciones de los estudiantes, se construyó una base de datos en el programa Excel 2013 para Windows, y posteriormente en el paquete estadístico IBM SPSS (C) Programa Estadístico para Ciencias Sociales, versión 19.0. Se analizaron los correspondientes promedios, frecuencias, y desviaciones estándar. Se aplicó la prueba de t-Student y la prueba exacta de Fischer, para identificar si había diferencias estadísticamente significativas $(p \leq 0,05)$, entre los promedios obtenidos en ambas modalidades. Se realizó una correlación a partir de la prueba de Pearson, entre cada uno de los estilos de aprendizaje con el rendimiento académico de la muestra de estudiantes, para estimar hasta qué nivel se relacionaron estas variables. La correlación se consideró significativa al nivel de 0,05 (bilateral).

\section{RESULTADOS Y ANÁLISIS}

En la Tabla 2 se observan los estilos de aprendizajes de los estudiantes que cursaron la asignatura de química durante los años 2012 y 2013. En la Tabla 2 se reparten el número de estudiantes que participaron por semestre en cada año escolar en las modalidades presencial y distancia, y al final se reporta el total de estudiante en cada modalidad con su respectiva puntuación. Se encontró que ambas modalidades estuvieron en la categoría alta $(\geq 8,0)$. En la modalidad a distancia estos valores fueron comparativamente mayores, variando de $10,13 \pm 0,35$ a $12,57 \pm 1,96$, mientras que en presencial oscilaron entre $7,97 \pm 2,87$ a $10,52 \pm 2,27$.

Tabla 2: Estilos de aprendizajes de los estudiantes de la asignatura de química en modalidades presencial (A) y a distancia (B)

\begin{tabular}{|c|c|c|c|c|c|c|c|c|c|c|}
\hline \multirow{3}{*}{$\begin{array}{c}\text { Estilo de } \\
\text { aprendizaje }\end{array}$} & \multicolumn{4}{|c|}{ Año 2012} & \multicolumn{4}{|c|}{ Año 2013} & \multicolumn{2}{|c|}{ Total } \\
\hline & \multicolumn{2}{|c|}{ Semestre I } & \multicolumn{2}{|c|}{ Semestre II } & \multicolumn{2}{|c|}{ Semestre I } & \multicolumn{2}{|c|}{ Semestre II } & \multirow{2}{*}{$\begin{array}{c}A \\
(n=41)\end{array}$} & \multirow{2}{*}{$\begin{array}{c}B \\
(n=72)\end{array}$} \\
\hline & $A(n=8)$ & $\begin{array}{c}B \\
(n=20)\end{array}$ & $\underset{(n=15)}{A}$ & $B(n=8)$ & $A(n=7)$ & $\begin{array}{c}B \\
(n=16)\end{array}$ & $\begin{array}{c}A \\
(n=11)\end{array}$ & $\begin{array}{c}B \\
(n=28)\end{array}$ & & \\
\hline Visual & $\begin{array}{c}8.88 \pm \\
2.17\end{array}$ & $\begin{array}{c}10.55 \pm \\
1.36\end{array}$ & $\begin{array}{c}9.00 \pm \\
1.60\end{array}$ & $\begin{array}{c}10.75 \pm \\
0.46\end{array}$ & $\begin{array}{c}10.02 \pm \\
2.24\end{array}$ & $\begin{array}{c}10.19 \pm \\
1.72\end{array}$ & $\begin{array}{c}8.55 \pm \\
2.02\end{array}$ & $\begin{array}{c}10.57 \pm \\
1.83\end{array}$ & $\begin{array}{l}9.11 \pm \\
2.01\end{array}$ & $\begin{array}{c}10.52 \pm \\
1.34\end{array}$ \\
\hline Auditivo & $\begin{array}{c}9.75 \pm 1 \\
58\end{array}$ & $\begin{array}{c}11.25 \pm \\
2.15\end{array}$ & $\begin{array}{c}8.80 \pm \\
2.47\end{array}$ & $\begin{array}{c}10.13 \pm \\
0.35\end{array}$ & $\begin{array}{c}8.43 \pm \\
3.26\end{array}$ & $\begin{array}{c}10.43 \pm \\
2.75\end{array}$ & $\begin{array}{c}8.64 \pm \\
2.80\end{array}$ & $\begin{array}{c}11.61 \pm \\
0.63\end{array}$ & $\begin{array}{c}8.91 \pm \\
2.53\end{array}$ & $\begin{array}{c}10.86 \pm \\
1.47\end{array}$ \\
\hline $\begin{array}{l}\text { Lectura- } \\
\text { Escritura }\end{array}$ & $\begin{array}{c}7.97 \pm \\
2.87\end{array}$ & $\begin{array}{c}11.79 \pm \\
1.15\end{array}$ & $\begin{array}{c}8.87 \pm \\
2.64\end{array}$ & $\begin{array}{c}12.18 \pm \\
1.96\end{array}$ & $\begin{array}{c}10.12 \pm \\
2.58\end{array}$ & $\begin{array}{c}11.61 \pm \\
2.24\end{array}$ & $\begin{array}{c}9.55 \pm \\
3.62\end{array}$ & $\begin{array}{c}12.57 \pm \\
2.13\end{array}$ & $\begin{array}{c}9.12 \pm \\
2.93\end{array}$ & $\begin{array}{c}12.04 \pm \\
1.05\end{array}$ \\
\hline Kinestésico & $\begin{array}{c}10.52 \pm \\
2.27\end{array}$ & $\begin{array}{c}12.29 \pm \\
0.75\end{array}$ & $\begin{array}{c}10.33 \pm \\
2.78\end{array}$ & $\begin{array}{c}12.35 \pm \\
1.70\end{array}$ & $\begin{array}{c}9.14 \pm \\
1.21\end{array}$ & $\begin{array}{c}11.97 \pm \\
1.55\end{array}$ & $\begin{array}{c}8.18 \pm \\
3.06\end{array}$ & $\begin{array}{c}11.87 \pm \\
2.15\end{array}$ & $\begin{array}{c}9.54 \pm \\
2.33\end{array}$ & $\begin{array}{c}12.12 \pm \\
1.47\end{array}$ \\
\hline
\end{tabular}

En la Tabla 3 se observan los resultados de la prueba t-student entre los estilos de aprendizajes de la muestra de estudiantes. Se puede ver que en la modalidad presencial, la varianza en cada estilo de aprendizaje fue más alta, al comparar con las obtenidas en la modalidad a distancia, presentándose diferencias significativas entre estos valores en ambas modalidades $(p<0,05)$. Por lo anterior se infiere que los estudiantes de ambas modalidades poseen diferentes maneras de aprender. 
En la Tabla 4, se puede ver el perfil de preferencia en los estilos de aprendizaje de los estudiantes consultados. Los estudiantes de distancia mostraron una marcada preferencia hacia los estilos kinestésico y lectura/escritura, mientras que los de la modalidad presencial reflejaron una tendencia hacia los estilos kinestésico, lectura/escritura y visual. Estos resultados permiten ampliar el enfoque general de los perfiles de aprendizaje de los estudiantes de Ingeniería Electrónica y Electromecánica que cursaron la asignatura de química. Se observa que se presentó una marcada preferencia hacia los estilos kinestésico $(32,5 \%)$ y lecto/escritor $(23,8 \%)$ en ambas modalidades, por lo que se puede afirmar que los estudiantes de estas carreras se les facilitan más los aprendizajes prácticos y experimentales de contacto con la realidad, así como la información mostrada en palabras. En menor proporción estuvieron los estilos visual $(8,9 \%)$ y auditivo (8,8\%). Así mismo, una parte importante de los estudiantes utilizó estilos de aprendizaje multimodales principalmente kinestésico-lecto/escritura (11,6\%) y tan solo el $4,0 \%$ utilizó la combinación de los cuatro estilos. Estos resultados fueron similares a los reportado por Valencia et al., (2012) en su trabajo sobre identificación de los perfiles de aprendizaje de estudiantes de tercer semestre de odontología, en el cual encontró estilos de aprendizaje diferentes, predominando el tipo visual, seguido por el auditivo y por último el kinestésico. Los resultados de este trabajo coinciden con los reportados por Ortiz et al., (2013) quien utilizó en cuestionario VARK en estudiantes de matemáticas y encontró preferencia con el kinestésico, seguido de auditivo y visual, aunque predominaron los estilos multimodales.

Tabla 3: Varianzas entre los estilos de aprendizaje por modalidad, $p<0,05^{*} ; p<0,01^{* *}$ y $p<0,005^{* * *}$

\begin{tabular}{|c|c|c|c|c|c|}
\hline Estilo & Presencial $(\mathrm{n}=41)$ & Distancia $(\mathrm{n}=72)$ & Valor crítico $\mathrm{t}$ (dos colas) & Estadístico $\mathrm{t}$ & $\mathrm{p}$ - valor \\
\hline Visual & 0.59 & 0.06 & 2.44 & -4.22 & $0.006^{\star \star}$ \\
\hline Auditivo & 0.44 & 0.18 & 2.44 & -4.31 & $0.003^{\star \star \star}$ \\
\hline Lectura/Escritura & 1.06 & 0.34 & 2.44 & -5.38 & $0.002^{\star \star \star}$ \\
\hline Kinestésico & 1.20 & 0.13 & 2.44 & -4.08 & $0.004^{\star \star \star}$ \\
\hline
\end{tabular}

Tabla 4: Perfil de preferencias en los estilos de aprendizaje de los estudiantes

\begin{tabular}{|l|c|c|}
\hline Perfil de aprendizaje & Presencial $(\mathrm{n}=41)$ & Distancia $(\mathrm{n}=72)$ \\
\hline Visual & 17.0 & 4.0 \\
\hline Auditivo & 12.0 & 7.0 \\
\hline Lectura/escritura & 20.0 & 26.0 \\
\hline Kinestésico & 23.0 & 38.0 \\
\hline Lectura/escritura - visual & 2.0 & 3.0 \\
\hline Kinestésico - auditivo & 5.0 & 3.0 \\
\hline Lectura/escritura - kinestésico & 8.0 & 14.0 \\
\hline Lectura/escritura - visual - auditivo & 7.0 & 2.0 \\
\hline Visual - auditivo - lectura/escritura - kinestésico & 6.0 & 3.0 \\
\hline Total & $100 \%$ & $100 \%$ \\
\hline
\end{tabular}

En la Tabla 5 se presentan los promedios de las calificaciones de los estudiantes que cursaron la asignatura de química durante los años 2012 y 2013. En la Tabla 5 se reparten el número de estudiantes que participaron por semestre en cada año escolar en las modalidades presencial y distancia. Se puede ver que los estudiantes de la modalidad a distancia obtuvieron un rendimiento global de $88,6 \%$ comparativamente más alto que el obtenido por los de presencial $78,4 \%$, lo que indica que las calificaciones de estudiantes en la modalidad a distancia fueron más altas y homogéneas.

Tabla 5: Promedios de las calificaciones modalidades presencial (A) y a distancia (B)

\begin{tabular}{|l|c|c|c|c|c|c|c|c|c|c|}
\hline \multirow{2}{*}{ Cohortes } & \multicolumn{4}{|c|}{2012} & \multicolumn{4}{c|}{2013} & \multicolumn{3}{|c|}{ Total } \\
\cline { 2 - 14 } & \multicolumn{2}{|c|}{ Semestre I } & \multicolumn{2}{|c|}{ Semestre II } & \multicolumn{2}{|c|}{ Semestre I } & Semestre II & \multicolumn{1}{|c|}{} \\
\hline Modalidad & $\mathrm{A}$ & $\mathrm{B}$ & $\mathrm{A}$ & $\mathrm{B}$ & $\mathrm{A}$ & $\mathrm{B}$ & $\mathrm{A}$ & $\mathrm{B}$ & $\mathrm{A}$ & $\mathrm{B}$ \\
\hline Número de estudiantes & 8 & 20 & 15 & 8 & 7 & 16 & 11 & 28 & 41 & 72 \\
\hline Calificación promedio & 3.65 & 4.43 & 4.04 & 4.47 & 3.81 & 4.29 & 4.16 & 4.53 & 3.92 & 4.43 \\
& \pm 0.54 & \pm 0.19 & \pm 0.39 & \pm 0.35 & \pm 0.35 & \pm 0.29 & \pm 0.45 & \pm 0.52 & \pm 0.60 & \pm 0.40 \\
\hline Mínimo & 2.7 & 4.1 & 3.0 & 3.9 & 3.0 & 3.5 & 3.0 & 3.9 & 2.7 & 3.5 \\
\hline Máximo & 4.4 & 4.9 & 5.0 & 5.0 & 4.6 & 4.9 & 4.8 & 5.0 & 5.0 & 5.0 \\
\hline Rango & 1.6 & 0.8 & 2.0 & 1.1 & 1.6 & 1.1 & 1.8 & 1.3 & 2.2 & 1.5 \\
\hline
\end{tabular}


Al aplicar la prueba exacta de Fischer, para analizar las calificaciones globales de los estudiantes de la asignatura de química, se observaron diferencias estadísticamente significativas entre las varianzas 0,32 (presencial) y 0,16 (distancia), valor $F(2,01)$ y $(p=0,00<0,05)$. En la Figura 1 , se detallan los promedios de las calificaciones de los estudiantes para cada actividad específica evaluada en la metodología, empleada en la asignatura en ambas modalidades durante los años 2012 y 2013 . Se resalta que en general, todos los estudiantes de química en la modalidad a distancia tuvieron un rendimiento académico más alto respecto a los de presencial, posiblemente por una mejor adaptación a la metodología aplicada por el docente. Los datos obtenidos al aplicar la prueba de Fischer indicaron que hubo diferencias estadísticamente significativas entre las calificaciones obtenidas por los estudiantes en las actividades de examen al inicio $(p=$ $0,03<0,05)$, proyecto de aula $(p=0,04<0,05)$, seminarios $(p=0,03<0,05)$, laboratorios $(p=0,04<0,05)$ y parcial final $(p=0,04<0,05)$. Mientras que en la calificación de los talleres independientes no hubo diferencias estadísticamente significativas $(p=0,38>0,05)$.

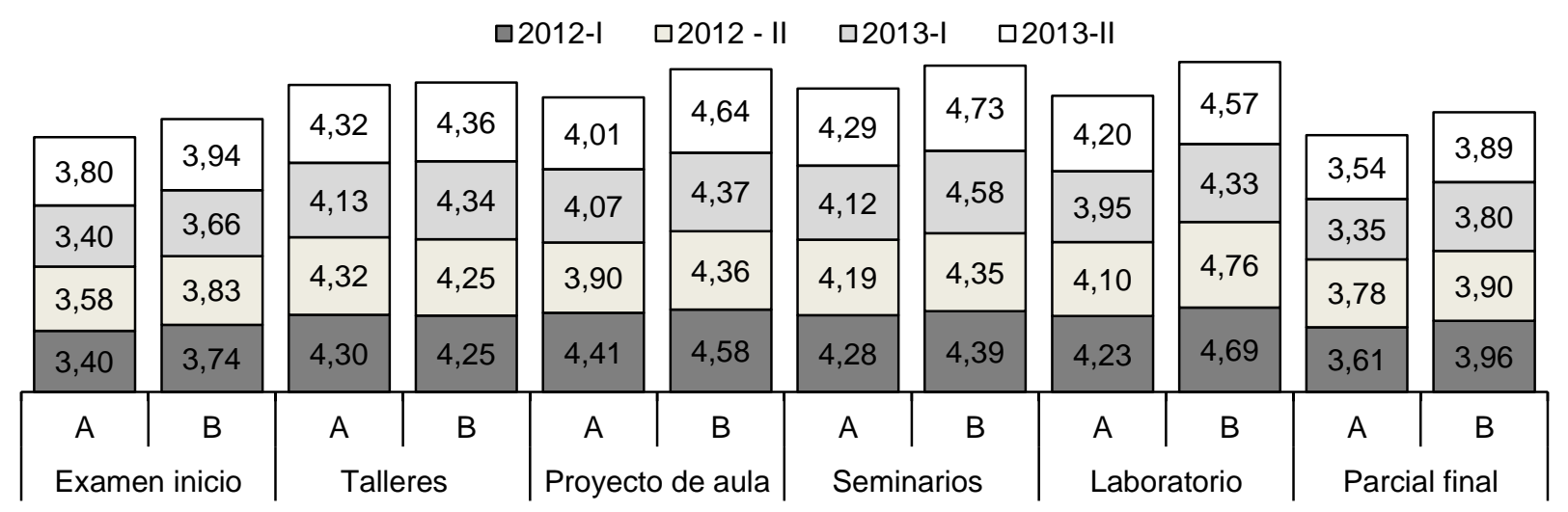

Fig. 1: Calificaciones globales de los estudiantes con la metodología empleada durante 2012 y 2013 , modalidades presencial $(A)$ y a distancia $(B)$.

En los estudiantes a distancia el promedio de edad fue mayor, por lo que estos pudieron haber tenido un grado de madurez emocional y personal diferente, un mayor compromiso y responsabilidad con la profesión, así como más independencia y organización propia. Otra variable importantísima a tener en cuenta es la actual ocupación y experiencia laboral de los alumnos a distancia, que son trabajadores, mayores, varios de ellos y a profesionales y que posiblemente se desempeñen en áreas afines a la química. Aguilar-Rivera (2010) afirman que el trabajo independiente y la madurez psicológica, fomenta en los estudiantes mayor sentido de responsabilidad y compromiso con sus actividades académicas, lo cual se refleja en la mayoría de los casos en un mejor rendimiento académico.

Cabe resaltar que el estudiante a distancia online es un personal capacitado que debe adaptarse a una nueva forma de estudio, que debe ser organizado, con capacidad de actualización y motivación. Esto es posible con disciplina, ilusión y una actitud que sepa recoger las ventajas de ser cada vez un poco más dueño de su tiempo. Blumen et al., (2011) afirma que la educación a distancia tiene como finalidad el aprendizaje a partir de la autoformación, donde el estudiante lleve la iniciativa y el docente sea un guía de dicho proceso. Por ello la metodología en la modalidad a distancia, tuvo un carácter más hacia lo práctico, donde se buscó que los estudiantes asumieran su propia formación en esta área de conocimiento, es decir se les dio más autonomía. Mientras que en la modalidad presencial a pesar que también se procuró que los estudiantes investigaran y tuvieran independencia con el trabajo fuera del aula, predominaron las clases magistrales teóricas donde fue el docente quien tomó la iniciativa.

Al analizar la procedencia de los estudiantes consultados en la Plataforma Educativa de Registro Académico de la IES, se resalta que en la modalidad presencial el $93 \%$ indicaron que eran bachilleres provenientes de la secundaria sin experiencia laboral y el 7\% confesaron poseer una formación técnica, tecnológica o profesional con experiencia en empresas industriales. Al compararlos con la modalidad a distancia, se observó una gran diferencia, ya que de estos, el 8\% solamente eran bachilleres sin experiencia laboral, mientras que 33\% eran técnicos, $42 \%$ tecnólogos, 17\% profesionales con experiencia laboral en entidades públicas y privadas de transformación o de servicios, así como en la industria del sector petroquímico, de plásticos, mecánica y en estudios de factibilidad y realización de proyectos. Por lo que pudieron haber tenido una visión más amplia de los conceptos manejados en la asignatura de química. Se destaca que no hubo mujeres que cursaron la asignatura durante el periodo de estudio.

Esguerra y Guerrero, (2010) en su estudio con estudiantes de psicología, indicaron que la edad y la experiencia profesional están asociadas con un mayor grado de independencia y organización personal, lo 
que impacta positivamente en el rendimiento académico de los estudiantes universitarios. Por su parte Montero-Rojas et al., (2007) aseguran que los factores asociados al rendimiento académico son diversos, ya que pueden ser de tipo personal, psicológico, familiar, o socioeconómicos, siendo este último uno de los más incidente. En general todos los estudiantes presentaron un menor rendimiento en los exámenes teóricos y mayor desenvolvimiento en las actividades prácticas y de interacción grupal, como los laboratorios, talleres independientes, seminarios y proyecto de aula, posiblemente porque estas últimas fueron actividades que se planificaron con anterioridad y en ellas los estudiantes se prepararon mejor investigando a fondo en los temas asignados, a su vez durante el transcurso de las mismas tuvieron la posibilidad del uso de herramientas tecnológicas e informáticas, que posiblemente les otorgó mayor confianza y potencializó sus habilidades. Elivir-Maiera (2004) encontró que el mayor rendimiento en estudiantes de medicina, se presentó en las revisiones bibliográficas, respecto a los exámenes sorpresivos, debido a que los estudiantes tuvieron mayor tiempo de preparación en estas. Feldman et al., (2008) indicó que el estrés está relacionado con el bajo rendimiento académico y recomendó que si los universitarios desean alcanzar sus logros, es indispensable que estén tranquilos, en confianza y dispuesto siempre a aprender.

En la Tabla 6, se detallan los valores obtenidos de las correlaciones entre el rendimiento académico y los estilos de aprendizajes de los estudiantes que cursaron la asignatura de química. En la parte superior se encuentran los datos de la modalidad a distancia, y se evidencia, que en estos no hubo correlación entre los estilos de aprendizaje y el rendimiento académico ( $p>0,05)$. Al comparar cada estilo se observó una correlación positiva y significativa entre los estudiantes que utilizaron el estilo visual con el auditivo ( $r=0,29$ y $p=0,04<0,05)$, con la lectoescritura $(r=0,34$ y $p=0,00<0,05)$ y con el kinestésico $(r=0,35$ y $p=0,00<0,05)$. Así mismo también se observó una relación directa entre los que emplearon el estilo auditivo con la lectoescritura.

Tabla 6: Correlación entre el rendimiento académico y los estilos de aprendizaje de los estudiantes que cursaron la asignatura de química. *La correlación es significante al nivel 0,05 (bilateral).

\begin{tabular}{|c|l|l|l|c|c|c|}
\hline \multicolumn{2}{|c|}{ Correlaciones } & $\begin{array}{l}\text { Rendimiento } \\
\text { Académico }\end{array}$ & Visual & Auditivo & $\begin{array}{c}\text { Lectura } \\
\text { Escritura }\end{array}$ & Kinestésico \\
\hline \multirow{2}{*}{$\begin{array}{c}\text { Rendimiento } \\
\text { Académico }\end{array}$} & Coeficiente de Pearson & 1 & $-0,01$ & 0,00 & $-0,03$ & 0,13 \\
\cline { 2 - 7 } & p-valor & & 0,94 & 0,96 & 0,79 & 0,28 \\
\hline \multirow{2}{*}{ Visual } & Coeficiente de Pearson & $-0,19$ & 1 & $0,29^{*}$ & $0,34^{*}$ & $0,35^{*}$ \\
\cline { 2 - 7 } & p-valor & 0,22 & & 0,04 & 0,00 & 0,00 \\
\hline \multirow{2}{*}{ Auditivo } & Coeficiente de Pearson & $-0,14$ & 0,09 & 1 & $0,26^{*}$ & 0,20 \\
\cline { 2 - 7 } & p-valor & 0,40 & 0,59 & & 0,03 & 0,09 \\
\hline \multirow{2}{*}{$\begin{array}{c}\text { Lectura } \\
\text { Escritura }\end{array}$} & Coeficiente de Pearson & $-0,13$ & 0,03 & 0,24 & 1 & 0,06 \\
\cline { 2 - 7 } K-valor & 0,43 & 0,86 & 0,13 & & 0,614 \\
\hline \multirow{2}{*}{ Kinestésico } & Coeficiente de Pearson & $-0,16$ & 0,25 & $-0,12$ & $-0,31^{*}$ & 1 \\
\cline { 2 - 7 } & p-valor & 0,31 & 0,11 & 0,47 & 0,04 & \\
\end{tabular}

En la parte inferior se detallan las correlaciones entre los estilos de aprendizaje de los estudiantes de la modalidad a presencial. Se observa que aunque hubo una escasa correlación negativa entre el rendimiento académico con cada uno de los estilos de aprendizaje, esta no fue significativa ( $p>0,05)$. Únicamente se observó una correlación inversa y significativa entre los estudiantes que utilizaron el estilo kinestésico con la lectoescritura ( $r=-0,31$ y $\mathrm{p}=0,04<0,05)$. Estos resultados fueron similares a los reportados por Ortiz et al., (2013) quien no encontró una relación estadísticamente significativa entre los estilos de aprendizaje visual, auditivo, lectoescritura y kinestésico con el rendimiento académico en estudiantes de matemáticas ( $>0,05)$. Estos utilizaron el cuestionario VARK, y señalaron que no hay ningún tipo de relación entre el rendimiento y los estilos de aprendizaje, a su vez indicaron que en el rendimiento académico pueden incidir otro tipo de factores: ambientales, físicos, motivacionales, emocionales, pero que es independientes del estilo de aprendizaje.

\section{CONCLUSIONES}

Los estudiantes que cursaron la asignatura de química presentaron diferentes formas de aprender $(p<0,05)$. Se les facilitó el aprendizaje empírico, así como con la información escrita. Todos los grupos presentaron una marcada preferencia hacia el estilo kinestésico $(32,5 \%)$. Los estudiantes a distancia se adaptaron mejor a la metodología aplicada, y presentaron mejores rendimientos académicos $(88,6 \%)$ en comparación con los presenciales $(78,4 \%)$. Lo anterior, posiblemente debido a la actual ocupación y experiencia laboral de los alumnos a distancia, que son trabajadores, mayores, varios de ellos y a profesionales y que posiblemente se desempeñen en áreas afines a la química. No se encontró correlación estadísticamente significativa entre los estilos de aprendizaje y el rendimiento académico en ambas modalidades $(p>0,05)$. La identificación del estilo de aprendizaje predominante de los estudiantes es una herramienta útil para adaptar la metodología de enseñanza del docente, a la vez que posibilita diseñar métodos de evaluación más apropiados para comprobar el progreso de los estudiantes. Se recomienda que en futuras investigaciones 
se usen muestras de estudiantes universitarios más comparables: con la misma edad, experiencia, metodología de enseñanza y características de rendimiento; o bien, que se evalúen aparte variables como: tipo de trabajo, número de años de experiencia y nivel de conocimientos previos de estos estudiantes, pues con ello se podrían explicar las diferencias encontradas en este trabajo. Además sería bueno analizar la influencia de la forma de evaluar y estilos de enseñanza del docente sobre el rendimiento académico de estos estudiantes.

\section{REFERENCIAS}

Aguilar-Rivera, M., Learning styles and learning strategies in university students, Revista de Psicología, 28(2), 1-8 (2010)

Aguilera, P.E., Los estilos de enseñanza, una necesidad para la atención de los estilos de aprendizaje en la educación universitaria, Revista Estilos de Aprendizaje, 10, 79-87 (2012)

Bahamón, M. y otros tres autores, Estilos y estrategias de aprendizaje, relacionadas con el logro académico en estudiantes universitarios, Pensamiento Psicológico, 11(1), 115-129 (2013)

Blumen, S., C. Rivero y D. Guerrero, Universitarios en educación a distancia: estilos de aprendizaje y rendimiento académico, Revista de Psicología, 29(2), 225-243 (2011)

Elvir-Mairena, J.R., Academic yield of medicine third year students with regard to diferent methologies, Rev Med Hond, 72 (2), 10-12 (2004)

Esguerra, G. y P. Guerrero, Estilos de aprendizaje y rendimiento académico en estudiantes de psicología, Divers Perspect Psicol., 6(2), 97-109 (2010)

Feldman, L. y otros cinco autores, Relationships between academic stress, social support, mental health and academic performance in Venezuelan university students, Universitas Psychologica, 7(3), 739-751 (2008)

Fleming, N.D. VARK: A Guide to Learning Styles. Visual, Auditory, Reading/Writing, and Kinesthesic, Inventory, 2007, [On-line] http://www.vark-learm.com. Acceso 24 de Diciembre (2014)

Gutiérrez, M., J. García y D. Vieira, Estudio de las variables que influyen en los estilos de aprendizaje de diferentes grupos de alumnos del grado magisterio de la Universidad de Valladolid, España, Revista Estilos de Aprendizaje: 10(10), 55-64 (2012)

Herrera, C.M. y C.P. Zapata, Estudio correlacional de estilos de aprendizaje de estudiantes con modalidad en ciencias naturales, Tecné, Episteme y Didaxis, 31, 27-43 (2012)

León-Castañeda, M.E. y otros cuatro autores, Profile of learning preferences of students and professors of medicine. Element to consider in teaching, Educación Médica, 13(1), 33-39 (2010)

Li, G., W. Chen y J. Duanmu, Determinants of International Students' Academic Performance A Comparison Between Chinese and Other International Students, J. of studies in Int. Education, 14(1), 389-405 (2010)

López-Aguado, M., Estilos de aprendizaje. Diferencias por género, curso y titulación, Revista Estilos de Aprendizaje, 7(7), 109-134 (2011)

Montero-Rojas, E., J. Villalobos-Palma y A. Valverde-Bermúdez, Institutional, pedagogical, psychosocial and socio-demographic factors related to academic performance at the University of Costa Rica, a multilevel analysis, Relieve, 13(2), 215-234 (2007)

Ortiz, E. L., Sánchez, A.L. y A. Lozano, Rea y estilos de aprendizaje según Vark en el aprendizaje de las matemáticas, Revista Internacional Magisterio, Educación y Pedagogía, 64, 91-93 (2013)

Sepúlveda-Carreño, M.J. y otros cinco autores, Diferencias de género en el rendimiento académico y en el perfil de estilos y de estrategias de aprendizaje en estudiantes de química y farmacia de la Universidad de Concepción, Review of Learning Styles, 7(7), 1-19 (2011)

Valencia, B.C., A. Contreras, y L. R. Hinojosa, identificación de los estilos de aprendizaje del modelo VAC en los alumnos del tercer semestre de odontología CICS-UST IPN, Revista de investigación del CICS-UST: 3(2), 1-22 (2012)

Ventura, A.C., Estilos de aprendizaje y prácticas de enseñanza en la Universidad. Un binomio que sustenta la calidad educativa, Perfiles Educativos, 33(Esp.), 142-154 (2011)

Willcox, M.R., Factores de riesgo y protección para el rendimiento académico: Un estudio descriptivo en estudiantes de Psicología de una universidad privada, Revista Iberoamericana de Educación, 55, 1-9 (2011) 